\title{
About some possibilities of diagnosing partial failures arising in complex agricultural machinery
}

\author{
Alexy Zotov ${ }^{1}$, Vadim Gritsenko ${ }^{1, *}$, and Andrey Gazizov ${ }^{1}$ \\ ${ }^{1}$ Don State Technical University (DSTU), 1, pl. Gagarina, 344002, Rostov-on-Don, Russia
}

\begin{abstract}
The article is devoted to the issue of partial failure in complex technical systems. The authors analyze the diagnostic procedure in agricultural machinery for various purposes, the results of which provide a diagram of a multi-level organization for assessing the condition of equipment. It is also proposed to expand the range of estimated diagnostic parameters for making a more reliable managerial decision. The introduction of expert systems and other similar intelligent systems (for example, expert decision support systems) for diagnosing complex malfunctions is justified.
\end{abstract}

\section{Introduction}

The range of machines and assemblies used in the agricultural industry is very wide and continues to expand. The most popular and common are tractors, trucks and combine harvesters. Their reliability characteristics largely determine the effectiveness of the agricultural industry, and therefore the welfare of society.

At the same time, the reliability of the main agricultural machines is the subject of special studies, since the features of their operation are easily visible, especially with narrow use, special approaches in the design and manufacture.

However, like any technical devices, agricultural machines are subject to various types of failures, the causes of which are varied, as are the identified signs of their manifestation.

State standards for reliability distinguish two types of failures that are different in consequences - full and partial. In the event of a complete failure, continued operation becomes possible only after recovery with the elimination of such a failure. Partial failure (FW) will be recognized as such in cases where the device where it occurred retains the ability to continue to work as intended, but possibly with a slight decrease in quality (Russian Standard 27.002-2015 Reliability in technology. Terms and definitions).

In PF, the task of making a decision on the continuation of functioning under certain conditions may arise. Such a decision requires a comprehensive justification with forecasts of possible consequences and the risks arising from it. [1,2]

In the subjective aspect, such a solution requires the use of excellent knowledge and analytics. An important role is also played by the fact that those who formulate recommendations and make decisions on the functioning of a actually faulty technical

*Corresponding author: vadim.gritsenko75@mail.ru 
device take some responsibility for possible consequences that are probabilistic in nature, which means that they may not always be interpreted objectively.

\section{Analysis of the issue of partial failure.}

The procedure for detecting PF compared with complete failure is more complex and has some features, namely, it is possible to fix PF only by analyzing various diagnostic signs and indicators. The signs characterizing the PF can be divided into the following types:

- Functional - these are the signs that most reflect deviations and violations associated with the performance of functions by individual systems, subsystems, devices, etc.

- Information - these are signs that are complexed by the observer and to the greatest extent characterize the change in the state parameters of the investigated object.

The following can be attributed to the difficulties of solving diagnostic problems on the basis of informational features of the PF:

a) failure is manifested, but the existing control system is not recorded;

b) there are signs of failure, recorded by the control system in readings that are not in the relevant technical documentation;

c) the diagnostic system produces a deliberately false result according to the place and type of failure (determined visually or tactilely);

d) the possibility of continued operation is being examined, but without risk assessment of such a decision.

In turn, the functional signs of PF allow:

a) heuristically determine the possibility of changing the operating mode without noticeable negative consequences;

b) characterize such a refusal as insignificant without apparent patterns of occurrence and disappearance;

c) to raise a question, if necessary, about a heavier operating mode in conditions when, in the lighter mode, the main functional parameters were at an extremely low level.

In turn, diagnostic signs can be divided into the following groups:

- formalizable - a group of indicators that can be measured by various measuring instruments and devices,

- non-formalizable - a group of indicators that cannot be measured, but can be recorded tactilely with the help of the senses.

It can be concluded that for the analysis of PF, not only the readings obtained from the metering devices of any parameters are subject to evaluation, but also parameters that cannot be measured must also be taken into account. [3]

Studies related to the behavior of technical objects in the conditions of the BS have shown that expert solutions with assessments of the situation require an analysis of the complex of tasks based on the following information:

- about the place, type and reason of the PF;

- about the mode of functioning at which the PF was manifested;

- on the degree of exposure to external factors;

- about individual features and characteristics of the object of analysis.

In addition, information on the status of supporting and interacting systems and subsystems, as well as on the level of professional training of staff, may be useful. [4] The data provided must contain information on the possibilities and time characteristics of operational maintenance, on the method of organizing routine maintenance and the time until the next inspection of the condition and the necessary repairs.

In complex technical complexes, when solving such problems, most of this information is provided by the systems of automatic and automated monitoring and diagnostics included 
in the composition, as well as programs for expert evaluations (based on the knowledge base) and decision support (based on the database). [5-8]

The implementation of the processes of diagnosis and decision making for the occurrence of PF can be represented in the form of a generalized algorithm, presented in Fig.1. It can be seen from the algorithm that the procedure for entering the PF is multistage, with the elimination of dangerous situations (for humans and equipment), emergency failures (with complete cessation of functioning), as well as highlighting the possible modes of further functioning and determining the recommended mode, taking into account the current situation, if necessary works and features of external influences.

From the point of view of the problems solved in the article, the operators 6, 8, 10 are of interest. The implementation of tasks and the achievement of the goals defined by these operators can occur at various levels, differing in competencies and capabilities of participants in the analysis of emerging situations. Be sure to keep in mind that those who are directly in interaction with the object and receive the primary information used for evaluations have the greatest information about the real situation. However, in difficult cases, the qualifications of these people may not be enough for the right decisions, on the other hand, when referring to specialists of higher qualifications and remote exchange, some of the information is lost. It is important to maintain a certain balance between the experience and knowledge of specialists and the information saturation of entry-level operators.

It is worth noting that the enterprise can operate machines that differ both in the types of functions performed (combine harvester, tractor, car, etc.) $[9,10]$, and in types of design. This leads to a complication of the choice of diagnostic method, however, this process can be simplified by maintaining and analyzing failure statistics in knowledge bases. [11]

In the machines and units of the agricultural complex, the introduction of complex computer systems for monitoring and diagnosis, and even more so expert evaluations or decision support, is not advisable for many reasons of an economic, reliable, operational, ergonomic nature. However, you can consider creating a distributed expert-consulting system in which the center is the center of expert assessments. This center can be unified not only for agricultural technology, but also for many similar devices in other industries. It is proposed to make such a system a multi-level, approximately implementing the principle of hierarchy, shown in Fig. 2.

$\mathrm{PF}$ are more diverse than complete ones, and, in addition, their diagnosis requires a certain amount of additional information, which is often missing due to the limited capabilities of control systems. Therefore, the tasks of higher levels will be more complicated due to the need to form conclusions in conditions of incomplete or fuzzy information about failures. There are a wide variety of causes of failures and remedial measures applied. [11] Typical diagnostic signs of PF are those parameters that are informal in nature and poorly measurable. For example: overheating, increased consumption of liquids, reduced power, weakening of mechanical gears, changes in transient control processes, violations of information interfaces, etc. The use of such information is possible, but the conclusions of expert assessments will be fuzzy in nature and evaluated in a probabilistic space. The preferred way to improve the quality of estimates, which means reducing the level of errors and associated losses, is an operational guided control in the interactive mode of the "operator - specialist - expert" system. [12-16] At the same time, the operator provides the results of objective (hardware) control, replenishing them (if necessary) with information on the failure received by visual and tactile means. The specialist identifies situations in which it is not possible to formulate a unique recommendation and, in this situation, makes a request to the expert. The expert makes an appropriate conclusion, using not only his own experience and knowledge, but also the ability to replenish or clarify diagnostic information in real time through the 
information channel. Moreover, an expert can use a replenished computer knowledge base and algorithms of a decision support system. The Technical Expertise Center delivers and updates the necessary software.

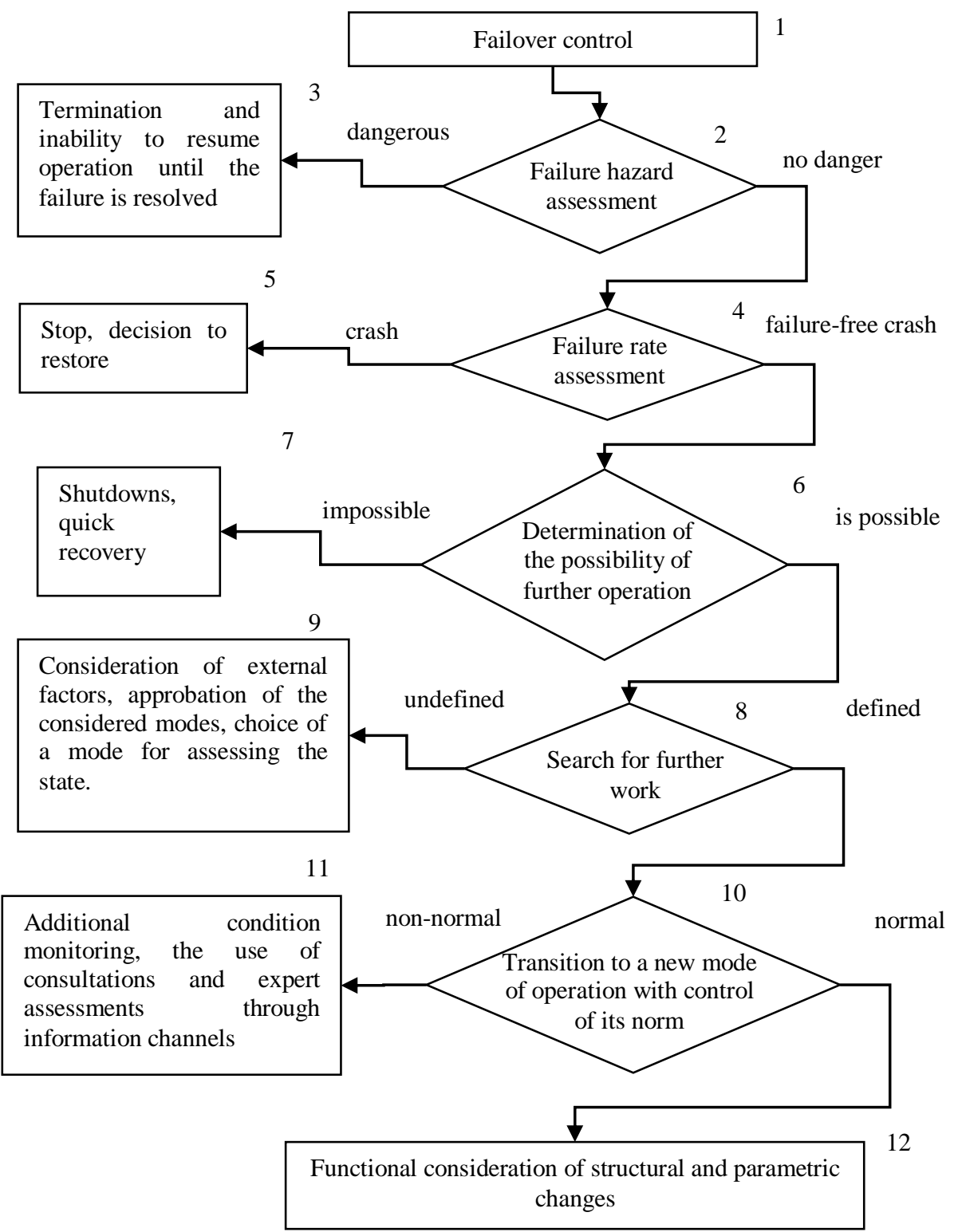

Fig. 1. Block diagram of a generalized decision-making algorithm for full and partial failures. 


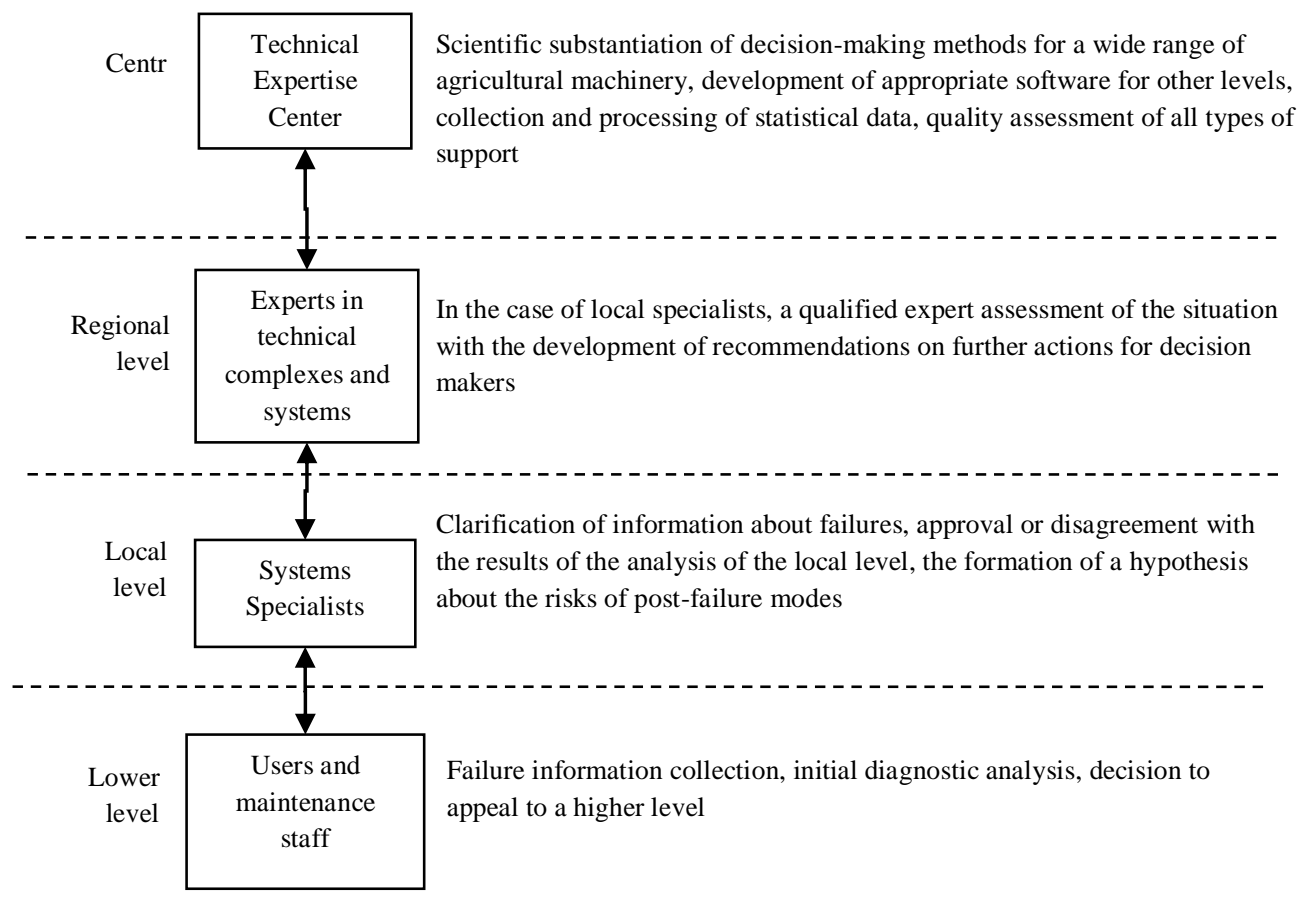

Fig. 2. Diagnostic Assessment Levels.

The rationale for such a system lies in the economic plane. Many foreign firms equip agricultural enterprises with imported equipment, which is distinguished by high productivity and sophisticated management, control, and diagnostic systems. [17] On-board computers are installed in the machines, the maintenance system works on the principle of the actual state, special algorithms for ensuring high efficiency are implemented.

However, along with this, it is becoming increasingly difficult to resolve the issues of establishing a reasonable balance between high productivity and temporary loss of recovery after emergencies. Some sources provide data on product losses due to the underutilization of the operational capabilities of modern agricultural machinery, reaching about $13-15 \%$ of the crop. [3] Major losses are due to downtime and sudden recovery. For some agricultural work, such losses are irretrievable (for example, due to weather conditions or sprinkling of grain).

The authors did not do economic calculations of the possible profitability of the proposals. At the same time, data are known that, for example, only in the Rostov region with a norm for the use of combine harvesters per 1000 ha equal to 6.02 pcs. with a total area of grain and grain crops equal to 2865 thousand hectares, technical support of almost 3 thousand combines, as well as a huge tractor fleet and other complex equipment is required. If we consider the entire Southern region, we will get even more significant figures characterizing the needs for organizing and solving agricultural problems. [18]

One of the authors put forward in the report of the proposals, witnessed the introduction of a close-to-purpose system in aviation. In the initial period, which lasted about a year, the system was inactive by users, there was some distrust of it. Subsequently, as the system improved and gained experience and knowledge, supporters appeared in the system, and then it took its rightful place in solving complex operational problems for various aircraft.

The theoretical rationale for the choice of decision-making strategies in the context of the appearance of private security is currently under development and does not have strict 
mappings in regulations (Russian Standard 27.310-95. Analysis of the types, consequences and criticality of failures. The main provisions).

\section{Conclusion}

The analysis of the possibilities of organizing the diagnosis of PF in agricultural machinery carried out in the article showed that the solution of this issue is possible with the use of expert systems, the result of which will be of a consultative nature. The expert system used provides recommendations on the most effective further operations in terms of current priorities, taking into account the specific situation and the admissibility of emerging risks.

The analysis of the possibilities for organizing the diagnosis of PF in agricultural machinery carried out in the article showed that the solution of this issue is possible using expert systems, the result of which will be of a consultative nature. The expert system used provides recommendations on the most effective further operational operations based on the current priorities of the manufacturing enterprise. The analysis of the public sector was carried out in general terms for a wide range of agricultural machinery, if we consider the diagnosis of a particular class of machines, then this will require additional research.

\section{References}

1. A.I. Zotov, V.V. Gritsenko, A.V. Cherpakov, Inženernyj vestnik Dona 4 (2018) ivdon.ru/uploads/article/pdf/IVD_156N4y18_Gricenko.pdf_935280522c.pdf

2. A.I. Zotov, V.V. Gritsenko, Inženernyj vestnik Dona 1 (2019) http://www.ivdon.ru/uploads/article/pdf/IVD_144_Zotov_N.pdf_5ce4a02e25.pdf

3. G. Craessaerts, J. De Baerdemaeker, W. Saeys, Biosystems Engineering 106, 26-36 (2010)

4. D.D. Bochtis, C.G.C. Sørensen, P. Busato, Biosystems Engineering 126, 69-81 (2014)

5. Z. Zhai, J. Fernán Martínez, V. Beltran, N. Lucas Martínez, Computers and Electronics in Agriculture 170 (2020)

6. B. Drury, R. Fernandes, M.-F. Moura, A. de Andrade Lopes, Information Processing in Agriculture 6, 487-501 (2019)

7. H. El Bilali, M. Sadegh Allahyari, Information Processing in Agriculture 5, 456-464 (2018)

8. R. Miodragović, M. Tanasijević, Z. Mileusnić, P. Jovančić, Expert Systems with Applications 39, 8940-8946 (2012)

9. D. Bochtis, C. Aage Gron Sorensen, D. Kateris, Operations Management in Agriculture, 79-115 (2019)

10. A. Ronkainen, IFAC Proceedings 46, 259-263 (2013)

11. Chen Chu, Zhao Zuo-xi, K.E. Xin-rong, Guo Yun-zhi, IFAC-PapersOnLine 51, 346352 (2018)

12. B.V. Pilev, Development of non-friction high-torque variators of agricultural machines (2013)

13. L.S. Guo, Q. Zhang, Biosystems Engineering 91, 261-269 (2005)

14. M. Kassler, Computers and Electronics in Agriculture 30, 237-240 (2001)

15. J.W. Jones, J.M. Antle, B. Basso and others, Agricultural Systems 155, 269-288 (2017)

16. S. O’Neill Somers, L. Stapleton, IFAC-PapersOnLine 48, 213-218 (2015) 
17. R.G. Berdnikova, Maintenance of tractors using an information support system (2013)

18. Development of technical equipment for agriculture in a market economy (FGBNU «Rosinformagrotech», Moscow, 2014) 